\title{
Measuring the Intensity Profile of Arbitrary Shaped Laser Foci Using Confocal Microscopy
}

\author{
Tobias Messer, Patrick Müller, and Martin Wegener
}

\begin{abstract}
D printing is an emerging field both on the macro and the nano scale. Especially while working in the nano scale, there are a lot of limiting factors for resolution. One way to improve it, is the usage of two-photon absorption in combination with the STED mechanism - well known from super-resolution microscopy. In this scheme, two lasers are used. One laser with a common Gaussian focus excites the photoresist via two-photon absorption, leading to polymerization, while a second laser with a different wavelength suppresses this reaction. The phase of the second laser is modified such way that a focus shape with a spot of zero intensity, e.g. a donut-shaped focus, arises. While not having a conceptual limit, the experimentally achievable resolution of this approach can be limited by shape deviations of the intensity profile of the second laser focus. Therefore, we are proofing the widely used method of measuring the profile via scattering from gold beads (100 nm size) by comparing it to measurements of the fluorescence signal of quantum dots (6 $\mathrm{nm}$ size) using confocal detection.
\end{abstract}

\footnotetext{
T. Messer ( $\triangle)$

Institute of Applied Physics, Karlsruhe Institute of Technology (KIT), Karlsruhe, Germany

e-mail: tobias.messer@student.kit.edu

P. Müller $\cdot$ M. Wegener

Institute of Applied Physics, Karlsruhe Institute of Technology (KIT), Karlsruhe, Germany

Institute of Nanotechnology, Karlsruhe Institute of Technology (KIT),

Eggenstein-Leopoldshafen, Germany 